\title{
Medical Image of the Week: Parietal Mass
}

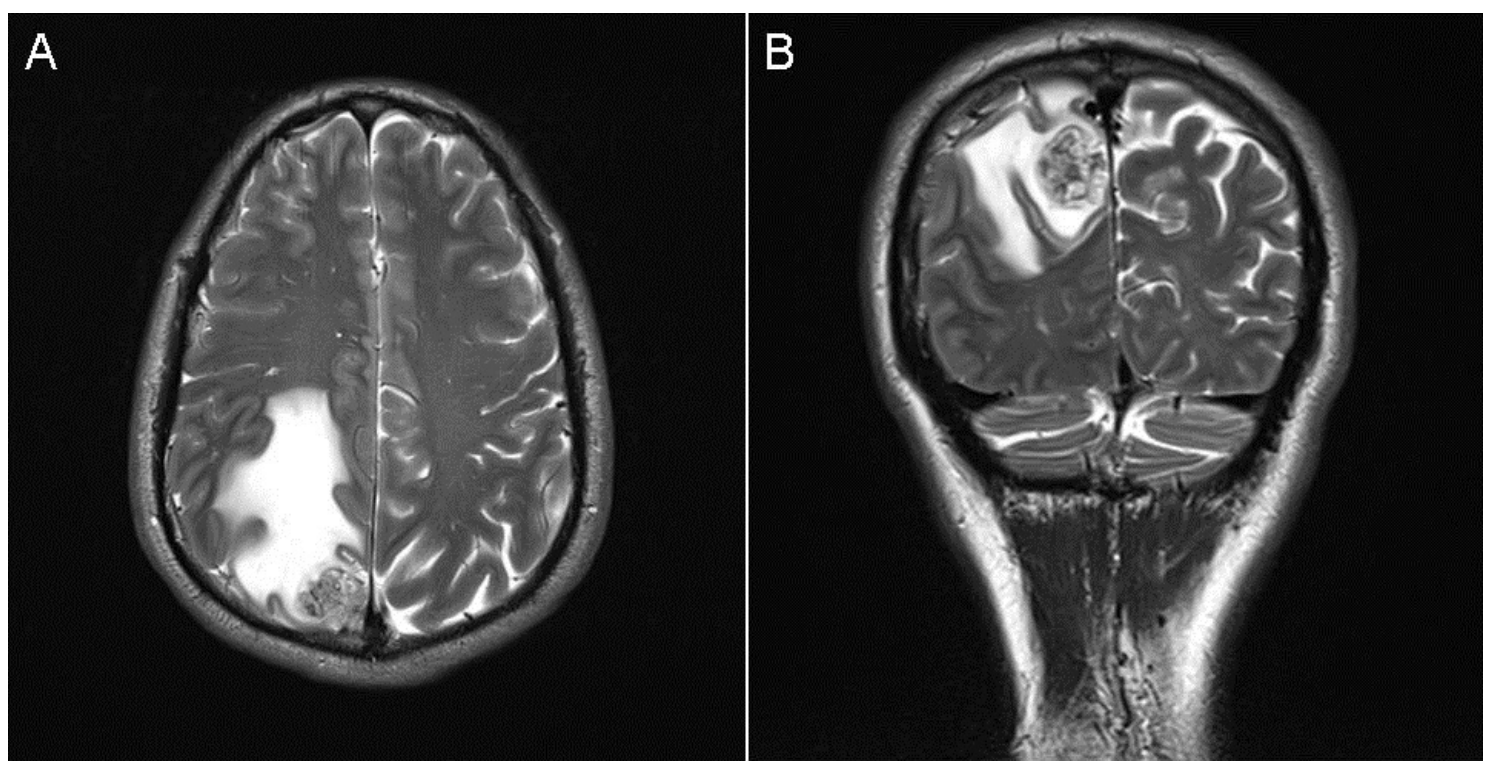

Figure 1. MRI of the head with contrast. Panel A: A $2.2 \mathrm{~cm} \times 2.1 \mathrm{~cm}$ enhancing mass within the right precuneus with surrounding vasogenic edema. Panel $B$ :

Coronal view.

A 48 year old man presented to the emergency department with a witnessed tonic clonic seizure by family members and 1 week worsening headaches and gait ataxia. Non-contrast $\mathrm{CT}$ of the head showed a large right-sided parietal mass. MRI of the head was done to further evaluate the mass (Figure 1). CT chest/abdomen/pelvis showed bilateral pulmonary nodules, mediastinal lymphadenopathy, hepatic lesions, and thickening of the mid-distal esophagus. The patient was evaluated by neurology and started on dexamethasone and levetiracetam. Neurosurgery was consulted and performed a right-sided craniotomy and parietal mass resection. Later, an EGD was performed and biopsies were taken of the esophagus. The patient was found to have metastatic esophageal adenocarcinoma.

Andrew I. Kovoor MD and Sudhir Kumar Tutiki MD

Department of Medicine

University of Arizona

Tucson, Arizona 